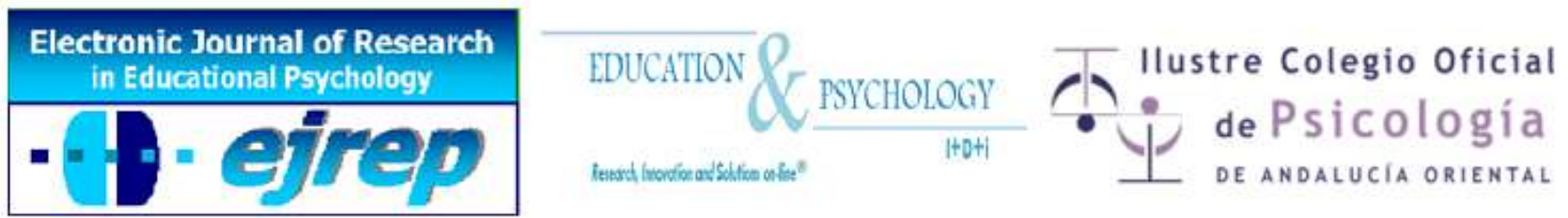

\title{
Entrenamiento cognitivo autoinstruccional para reducir el estilo cognitivo impulsivo en niños con el Trastorno por Déficit de Atención con Hiperactividad
}

\section{Gladys Wilma Rivera Flores ${ }^{1}$}

Programa Profesional de Psicología, Universidad Católica de Santa María, Arequipa-Perú

\section{Perú}

Correspondencia: Gladys Wilma Rivera Flores. Gral. Sánchez Cerro 318. Manuel Prado-Paucarpata. ArequipaPerú. E-mail: gladys_wrf@yahoo.es

(C) Education \& Psychology I+D+i and Ilustre Colegio Oficial de Psicólogía de Andalucía Oriental 


\section{Resumen}

Introducción. Los niños con el trastorno por déficit de atención con hiperactividad (TDAH) presentan un estilo cognitivo impulsivo, rígido y dependiente del campo. En este estudio se examina si el entrenamiento cognitivo autoinstruccional reduce el estilo cognitivo impulsivo de los niños con este trastorno.

Método. Los participantes fueron 10 niños de 6 a 8 años de edad diagnosticados con el trastorno por déficit de atención con hiperactividad (TDAH). Se empleó el diseño cuasiexperimental con pretest-posttest. La variable independiente fue el entrenamiento cognitivo autoinstruccional que consistió en 30 sesiones grupales dirigidas a 3 grupos conformados según la edad cronológica de los participantes. La variable dependiente fue el estilo cognitivo impulsivo, el cual fue evaluado antes y después del entrenamiento mediante el Test de Emparejamiento de Figuras Conocidas.

Resultados. El análisis pretest-posttest refleja una disminución estadísticamente significativa del percentil de impulsividad y el número de errores y un aumento estadísticamente significativo de la latencia después del entrenamiento.

Discusión y Conclusión. Los resultados demuestran la efectividad del entrenamiento cognitivo autoinstruccional para reducir el estilo cognitivo impulsivo del TDAH. A través del entrenamiento autoinstruccional, los niños aprendieron a hablarse a sí mismos una secuencia de pensamientos organizados para resolver tareas cognitivas de forma reflexiva.

Palabras Clave. Trastorno por déficit de atención con hiperactividad, estilo cognitivo, reflexividad-impulsividad, entrenamiento cognitivo autoinstruccional. 


\title{
Self-instructional cognitive training to reduce impulsive cognitive style in children with Attention Deficit with Hy- peractivity Disorder
}

\begin{abstract}
Introduction. Children with attention deficit hyperactivity disorder (ADHD) have an impulsive, rigid and field-dependent cognitive style. This study examines if the self-instructional cognitive training reduces the impulsive cognitive style of the children diagnosed with this disorder.

Method. The subjetcs were 10 children between the age of 6 and 8 years old diagnosed with attention deficit hyperactivity disorder (ADHD). The research design was quasi-experimental with pretest-posttest. The independent variable was the self-instructional cognitive training which consisted of 30 group sessions applied to 3 groups formed according the age of the subjects. The dependent variable was the impulsive cognitive style which was tested before and after the training with the Matching Familiar Figures Test.

Results. The analysis pretest-posttest shows a statistically significant reduction of the percentile impulsivity and the number of errors and a statistically significant increase of latency after the training.

Discussion and Conclusion. The results show the effectiveness of the self-instructional cognitive training to reduce the impulsive cognitive style of ADHD. Through self-instructions training, children learned to talk to themselves a sequency of organized thoughts that let them solve cognitive tasks in a reflective style.
\end{abstract}

Keywords: Attention deficit hyperactivity disorder, cognitive style, reflection-impulsivity, self-instructional cognitive training.

Reception: 07/08/14 Initial acceptance: 10/23/14 Final acceptance: 03/10/15 


\section{Introducción}

El trastorno por déficit de atención con hiperactividad (TDAH) es un trastorno debido a un retraso en el desarrollo neuropsicológico que provoca disfunciones en los mecanismos de control ejecutivo e inhibición del comportamiento, afectando de forma directa a los procesos psicológicos del sistema ejecutivo (memoria de trabajo y su relación con el sistema de atención anterior, la autorregulación de la motivación y el afecto, la internalización del lenguaje y los procesos de análisis y síntesis) y a sus funciones ejecutivas como planificación/organización, flexibilidad, automonitorización/evaluación implicadas directamente en las tareas de enseñanza y aprendizaje y en la adaptación escolar, familiar y social (Lavigne y Romero, 2010).

El TDAH se caracteriza por un patrón persistente de inatención y/o hiperactividad/impulsividad que interfiere con el funcionamiento o desarrollo (American Psychiatric Association, 2013). El trastorno es hereditario y además existen factores de riesgo durante el embarazo y el nacimiento (Larsson, Chang, D'Onofrio y Lichtenstein, 2014; Ketzer, Gallois, Martinez, Rohde y Schmitz, 2012; Rivera-Flores, 2013) y tiene como base fisiopatológica una disfunción del circuito frontoestriatal que involucra la corteza prefrontal y a su relación con los núcleos de la base, tálamo y cerebelo (Fernández-Mayoralas, Fernández-Jaén, GarcíaSegura y Quiñones-Tapia, 2010). El TDAH se presenta en alrededor del $5 \%$ de niños y $2.5 \%$ de adultos en la mayoría de culturas y los síntomas de inatención, hiperactividad impulsividad ocurren antes de los 12 años (American Psychiatric Association, 2013).

La inatención se manifiesta en conductas como estar fuera de la tarea, carecer de persistencia, tener dificultad para mantener la atención y ser desorganizado; estas conductas no se deben a actitudes desafiantes o falta de entendimiento. La hiperactividad se refiere a la actividad motora excesiva en situaciones en las que es inapropiado manifestarla, estar inquieto o hablar en exceso. La impulsividad consiste en actuar apresuradamente sin pensar en las consecuencias posteriores y poniendo en riesgo su propia integridad (ejemplo, lanzarse a la calle sin mirar), con frecuencia se entrometen en juegos, actividades o conversaciones de otros. La impulsividad refleja un deseo de recompensa inmediata o una incapacidad para postergar la gratificación (American Psychiatric Association, 2013). 


\section{Estilos cognitivos}

Los estilos cognitivos son definidos como estrategias habituales y estables que determinan los modos de percibir, recordar, pensar y solucionar problemas (Ramiro, Navarro, Menacho y Aguilar, 2010). Consisten en modos personales de procesamiento y estrategias para enfrentarse a la resolución de tareas determinadas (López, Serrano, Delgado, De Llano, Sánchez y Alberola, 2011). En la investigación de López, Serrano, Delgado, De Llano, Sánchez y Alberola (2011) se comparó los estilos cognitivos de niños con y sin TDAH; los niños con TDAH mostraron mayor impulsividad, mayor rigidez y más dependencia de campo. Otros investigadores (López, 2005; Orjales, 2002a) llegaron a las mismas conclusiones.

La dimensión cognitiva reflexividad-impulsividad surge con las investigaciones de Kagan y colaboradores en los años sesenta (Cairns y Cammock, 2005) y hace referencia a la preferencia mostrada por las personas para responder rápidamente versus quien prefiere hacerlo de forma pausada para reducir el número de errores en tareas de resolución de problemas (Ramiro, Navarro, Menacho y Aguilar, 2010; Bornás, Servera, Serra y Escudero, 1990). La reflexividad-impulsividad se operacionalizó a partir de la medida de dos componentes, latencia de respuesta y errores en el Test de Emparejamiento de Figuras Familiares (MFF) (Bornás, Servera, Serra y Escudero, 1990). Este test ha sido utilizado en diferentes estudios (Orjales y Polaino-Lorente, 1992; López, Serrano, Delgado, De Llano, Sánchez y Alberola, 2011; López, Serrano, Delgado, De Llano, Alberola, Sacristán, Pérez y Camina, 2010; Calderón, 2001; Arco, Fernández e Hinojo, 2004; Mateo, 2006) para evaluar la dimensión cognitiva reflexividad-impulsividad en niños con TDAH.

Los niños reflexivos emplean más tiempo para responder y cometen un número bajo de errores (López, Serrano, Delgado, De Llano, Alberola, Sacristán, Pérez y Camina, 2010; Ramiro, Navarro, Menacho y Aguilar, 2010), tienen control de los impulsos, realizan comprobación de hipótesis y análisis sistemático (López, Serrano, Delgado, De Llano, Sánchez y Alberola, 2011). Mientras que los niños impulsivos cometen más errores y responden más rápidamente (Ramiro, Navarro, Menacho y Aguilar, 2010) debido a la pobreza en los procesos de percepción y en el análisis de la información (López, Serrano, Delgado, De Llano, Sánchez y Alberola, 2011; Orjales, 2002a).

Sin embargo, el tiempo antes de la respuesta no únicamente determina el estilo cognitivo impulsivo-reflexivo, porque al intentar enseñar a los impulsivos a aumentar el tiempo 
antes de la respuesta, ellos seguían cometiendo errores y los reflexivos, por otro lado, tendrían éxito no porque hacen la tarea más despacio sino porque durante ese tiempo antes de la respuesta ellos utilizan estrategias cognitivas efectivas de procesamiento de información que finaliza en la solución correcta de los problemas (Clariana, 1993).

En efecto, existen diferencias en las estrategias del procesamiento de la información utilizadas por los impulsivos y los reflexivos. Los niños impulsivos responden al azar y de forma poco sistemática porque carecen de estrategias de solución de problemas. Los niños impulsivos obtienen información incompleta y responden intuitivamente sin pensar porque se saltan pasos importantes del modelo apropiado de solución de problemas como observar, acopiar la información, analizar, desechar datos innecesarios, trazar un plan de acción, tantear todas las posibilidades de respuesta, predecir las posibles consecuencias de cada respuesta, tomar una decisión, comprobar el resultado, felicitarse si la respuesta es correcta o analizar de nuevo la información y todas las alternativas si la respuesta es incorrecta (Orjales, 2002a).

Los reflexivos utilizan una estrategia analítica o de detalle, caracterizada por el mantenimiento de la atención y la valoración diferencial de todas las posibilidades de respuesta, es decir analizan los diferentes componentes de la tarea en base a comparaciones homólogas utilizando un proceso exhaustivo y sistemático; además están motivados para resolver bien problemas de tipo intelectual y tienen buenas estrategias de recuerdo (Clariana, 1993). Las investigaciones de Kagan, Rosman, Day, Albert y Phillips (1964) sostienen que existen dos disposiciones cognitivas en la producción de conceptos analíticos: a) la tendencia a reflexionar sobre todas las alternativas de solución en situaciones en las que están disponibles de forma simultánea varias alternativas de respuesta y b) la tendencia a analizar las alternativas en las partes que la componen; existiendo una relación inversa entre la producción de conceptos analíticos y los grados extremos de hiperactividad y distractibilidad.

\section{Entrenamiento cognitivo autoinstruccional}

El tratamiento de la impulsividad tuvo éxito con la aplicación del entrenamiento cognitivo autoinstruccional de Meichenbaum y Goodman (1971) que requería que el niño impulsivo se hable a sí mismo, inicialmente en voz alta y luego en forma de habla internalizada en un intento de incrementar el autocontrol. Las primeras aplicaciones del entrenamiento autoinstruccional fue con población de niños impulsivos e hiperactivos (Meichenbaum, 1977) y 
tenía por objetivo reducir la impulsividad (Bornás, Servera, Serra y Escudero, 1990; Meichenbaum, 1977).

Para proponer el entrenamiento cognitivo autoinstruccional, Meichenbaum fue influenciado por los estudios de Luria acerca del habla interna que muestra una secuencia en la que inicialmente la conducta del niño es controlada por las verbalizaciones externas de otros (adultos), luego el niño produce sus verbalizaciones en voz alta y finalmente a la edad de $5 \mathrm{o}$ 6 años, el niño controla su conducta por medio de autoverbalizaciones internas. Una segunda influencia para Meichenbaum fueron las investigaciones acerca de las estrategias cognitivas mediacionales en los niños, en las que se demostró que las estrategias autogeneradas como las autoinstrucciones y/o autoalabanzas (decir una oración particular o pensar en un pensamiento concreto) utilizadas mientras se realiza una tarea reducen la frustración que genera el retraso de la gratificación y ayuda a regular la conducta (Kendall y Braswell, 1993).

Los niños con TDAH presentan dificultades de autohablarse en tareas de resolución de problemas y en la autocreación de reglas para guiar la conducta, en especial cuando la conducta debe ser organizada en una larga cadena de acciones ordenadas jerárquicamente y en aquellas en las que el habla internalizada permitiría utilizar reglas o instrucciones aprendidas en el pasado (Barkley, 1997; citado por Orjales, 2002a).

Para ayudar a frenar y organizar el pensamiento de los niños impulsivos, Meichenbaum (1977) consideró necesario pedirles que verbalizaran en voz alta sus pensamientos para después ayudarles a organizarlos siguiendo una serie de pasos que denominó autoinstrucciones con el objetivo de interrumpir la cadena de pensamientos incorrectos que el niño impulsivo elabora momentos antes de la solución de un problema (prepotent responses). Mediante el aprendizaje de las autoinstrucciones, los niños impulsivos sustituían los pensamientos desorganizados por una secuencia de pensamientos útiles para la solución de problemas.

En el entrenamiento en mediación cognitiva o autoinstruccional de Meichenbaum, la mediación verbal consiste en hablar con uno mismo, de manera pertinente, cuando se enfrenta con algo que desea aprender, un problema que quiere resolver, o un concepto que quiere alcanzar (Polaino-Lorente, Avila, Cabanyes, García, Orjales y Moreno, 1997). 
Las conclusiones de investigaciones realizadas sobre el control verbal en el laboratorio y los estudios observacionales sobre el habla privada y la resolución de problemas demuestran que los niños impulsivos no tienen el hábito de analizar de forma espontánea sus experiencias en términos mediacionales cognitivos, no formulan ni internalizan reglas que los guíen en nuevas situaciones de aprendizaje; es decir, los niños impulsivos no comprenden la naturaleza del problema o tarea, entonces no pueden descubrir que mediadores utilizar (deficiencia en la comprensión), pueden tener un repertorio interno de mediadores cognitivos pero fallan en producirlos de forma adecuada y espontánea (deficiencia en la producción); además, los mediadores que el niño produce no guían la conducta que se está llevando a cabo (deficiencia mediacional). El objetivo de las autoinstrucciones es enseñar a los niños impulsivos a comprender la tarea, producir espontáneamente mediadores y estrategias y utilizarlos para guiar, monitorear y controlar su desempeño y de esta manera mejorar el proceso cognitivo compuesto por los estadios de comprensión, producción y mediación (Meichenbaum, 1977).

Meichenbaum describe que en el primer nivel del entrenamiento el terapeuta o tutor modela conductas que conducen a la solución exitosa de una tarea mientras habla a sí mismo en voz alta las instrucciones que guian su conducta hacia la solución de la tarea. Estas verbalizaciones (autoinstrucciones) son específicas a la tarea e incluyen oraciones que definen el problema (por ejemplo, clarificar y comprender los exactos requerimientos de la tarea), aproximación al problema (planear una estrategia general para solucionar el problema), enfocar la atención, seleccionar una respuesta y autorreforzarse por la ejecución correcta o utilizar una oración para motivarse y enfrentar la incorrecta ejecución. Después de observar al terapeuta ejecutando diferentes tareas, el niño ejecuta una tarea mientras habla a sí mismo en voz alta. Usualmente en este punto el terapeuta ayuda al niño a recordar la secuencia modelada de autoverbalizaciones. El terapeuta y el niño frecuentemente se alternan para ejecutar las tareas y conforme avanzan, el terapeuta va susurrando estas autoverbalizaciones y motivando al niño a que haga lo mismo, luego, tanto el terapeuta y el niño utilizan autoverbalizaciones encubiertas para controlar y dirigir el desempeño de la tarea. Entonces el entrenamiento autoinstruccional incluye el entrenamiento en el uso de verbalizaciones relacionadas directamente con la tarea, oraciones de autorrefuerzo y modelamiento de conductas apropiadas para la tarea (Kendall y Braswell, 1993).

Orjales (2007) adapta el esquema de Meichenbaum y Goodman de 1971 (pasos 2 al 6) y lo amplía agregando la primera autoinstrucción "Primero miro y digo todo lo que veo" (pa- 
so 1) dando lugar a 6 autoinstrucciones que ayudan a los niños con TDAH a disminuir la impulsividad cognitiva:

- Paso 1: Primero, miro y digo todo lo que veo.

- Paso 2: ¿Qué és lo que tengo que hacer?

- Paso 3: ¿Cómo lo voy a hacer?

- $\quad$ Paso 4: Tengo que estar muy atento (y ver todas las posibilidades de respuesta)

- Paso 5: ¡Ya puedo hacerlo!

- Paso 6: No me ha salido bien ¿Por qué? (repaso todos los pasos). Ah, ¡era por eso!. Bien la próxima vez no cometeré ese error.

De acuerdo a Meichenbaum (1977) el procedimiento de administración individual del entrenamiento cognitivo autoinstruccional es el siguiente:

1. Un adulto que hace de modelo, ejecuta una tarea hablándose a sí mismo en voz alta (modelado cognitivo).

2. El niño ejecuta la misma tarea siguiendo las instrucciones del modelo (guía externa abierta).

3. El niño ejecuta la tarea mientras habla en voz alta las instrucciones (autoguía abierta).

4. El niño susurra las instrucciones a sí mismo mientras realiza la tarea (autoguía abierta).

5. El niño realiza la tarea mientras guía su desempeño a través del habla privada (autoinstrucciones encubiertas).

Las autoinstrucciones potencian el pensamiento secuencial con el objetivo de facilitar la comprensión de las situaciones, generar las estrategias y mediadores necesarios para su resolución y utilizar tales mediadores en la guía y control de su comportamiento (Miranda y Soriano, 2010).

\section{Objetivo e hipótesis}

De acuerdo a lo expuesto anteriormente, el objetivo de esta investigación fue determinar si el entrenamiento cognitivo autoinstruccional disminuía el estilo cognitivo impulsivo que los niños con TDAH tienen para resolver tareas cognitivas. Este objetivo fue denifido en la siguiente hipótesis de investigación: el entrenamiento cognitivo autoinstruccional dismi- 
nuirá el estilo cognitivo-impulsivo porque el aprendizaje de las autoinstrucciones permite a los niños con TDAH resolver de forma reflexiva y sistemática las tareas cognitivas.

\section{Método}

\section{Participantes}

Diez niños diagnosticados con TDAH, de 6 a 8 años de edad, nacidos en Arequipa (Perú), conformados por 9 niños y 1 niña procedentes de colegios particulares. Todos los niños cumplieron los siguientes criterios de inclusión:

1) Un percentil de 77 o mayor en el cociente total del TDAH, medido por el Test para el trastorno por déficit de atención/hiperactividad de Gilliam (1995), escala aplicada a padres.

2) Un percentil de 86 o mayor en el estilo cognitivo impulsivo medido por el Test de Emparejamiento de Figuras Conocidas-20 de Cairns y Cammock (2005).

3) Un CI igual o superior a 90 medido por el WISC-IV (Weschler, 2005).

4) La ausencia de cualquier otro trastorno de origen orgánico o psicopatológico.

5) Con medicación.

Debido a que el entrenamiento cognitivo autoinstruccional se aplicó de forma grupal, los niños fueron distribuidos en 3 grupos de tratamiento de acuerdo a la edad cronológica. Un grupo de 3 niños de 6 años, un grupo de 4 niños de 7 años y un grupo de 3 niños de 8 años de edad. Cada grupo participó de forma grupal en el entrenamiento cognitivo autoinstruccional.

\section{Instrumentos}

Se utilizó el Test de Emparejamiento de Figuras Conocidas MFF-20 (Cairns y Cammock, 2005). Consiste en una prueba de emparejamiento perceptivo para medir el estilo cognitivo reflexivo-impulsivo. Bajas puntuaciones corresponden a un estilo cognitivo reflexivo (latencia larga y disminución en el número de errores) y altas puntuaciones corresponden a un estilo cognitivo impulsivo (latencia corta y aumento en el número de errores). El test está conformado por un total de 20 ítems de evaluación y 2 ítems iniciales de ensayo. Cada ítem se caracteriza por la presencia simultánea de un dibujo modelo, familiar para el niño (lentes, oso, casa) y 6 opciones diferentes de respuesta. La tarea del sujeto evaluado consiste en buscar 
entre las 6 opciones de respuesta, aquella opción que es exactamente igual al modelo. La aplicación del test es individual, dirigido a niños de 6 a 12 años y dura aproximadamente de 15 a 20 minutos.

El MMF-20 presenta valores de consistencia interna (alfa de Cronbach) entre 0.92 y 0.98 para las latencias $(\mathrm{M}=0.95)$, así como entre 0.68 y 0.82 para los errores $(\mathrm{M}=0.76)$. La validez teórica de la prueba refleja elevadas correlaciones negativas entre latencia y errores ( $\mathrm{r}$ $=-0.65)$. El estudio de la consistencia temporal con niños entre 6 y 9 años (estudiados en un periodo de dos años) presenta valores de coeficientes de consistencia longitudinal para niños entre 0.42 y 0.61 en latencias, así como entre 0.72 y 0.92 para errores; las niñas presentan coeficientes entre 0.17 y 0.44 para latencias, así como entre 0.61 y 0.97 para errores. El MMF-20 presenta coeficientes de consistencia longitudinal para la impulsividad entre 0.46 y 0.61 para los niños, así como entre 0.46 y 0.56 para niñas (Buela-Casal, Carretero-Dios, De los Santos-Roig y Bermúdez, 2003; citado por López, Serrano, Delgado, De Llano, Alberola, Sacristán, Pérez y Camina, 2010).

\section{Procedimiento}

El total de la muestra de niños con TDAH participó del programa de entrenamiento para descifrar instrucciones escritas de Orjales (2002b), el cual está basado en el entrenamiento cognitivo autoinstruccional de Meichenbaum y Goodman (1971) y el esquema autoinstruccional ampliado por Orjales (2007).

El programa de entrenamiento para descifrar instrucciones escritas tiene por objetivo reforzar la autoinstrucción ¿Qué es lo que tengo que hacer?; además forma parte de un programa de entrenamiento más amplio, el entrenamiento para reducir la impulsividad y el desarrollo de las estrategias de la solución de problemas (Orjales, 2002b).

El modelo ampliado de autoinstrucciones de Orjales (2007) utilizado en la investigación consta de 6 autoinstrucciones o verbalizaciones modeladas grupalmente (al inicio el terapeuta habla las autoinstrucciones en voz alta, luego los niños las hablan en voz alta para sí mismos, luego los niños las susurran y luego utilizan el habla interna). Las autoinstrucciones fueron las siguientes:

- "Primero miro y digo lo que veo" (autoinstrucción 1) entonces, los niños describen de forma precisa y bien organizada todos los elementos que componen la tarea. 
- “¿Qué es lo que tengo que hacer” (autoinstrucción 2) "eso lo pone en la parte escrita”.

- “¿Cómo lo voy a hacer?” (autoinstrucción 3) "leyendo por partes”.

- “Tengo que estar muy atento" (autoinstrucción 4).

- Dicen cada instrucción relativa a la tarea y resuelven parte a parte.

- “¿Me ha salido bien?” (autoinstrucción 5).

- Corrección en grupo del ejercicio: análisis de las alternativas propuestas por cada niño, evaluación de pros y contras de cada una de ellas, elección de la alternativa adecuada.

- "Me salió bien"/ "Me ha salido mal ¡ah era por eso! La próxima vez no cometeré ese error" (autoinstrucción 6).

Además, para enseñar las autoinsrucciones se utilizó el procedimiento diseñado por Meichenbaum (1977), cuidando que las verbalizaciones modeladas grupalmente siguieran la siguiente secuencia conforme avanzaban a lo largo del programa de entrenamiento:

1) El terapeuta modela la tarea mientras habla para sí mismo.

2) Los niños realizan la tarea mientras el terapeuta dirige en voz alta paso a paso cada autoinstrucción y las instrucciones específicas de cada tarea.

3) Los niños realizan la tarea hablando en voz alta para sí mismos las autoinstrucciones e instrucciones específicas de cada tarea mientras el terapeuta susurra las autoinstrucciones.

4) Los niños realizan la tarea susurrando suavemente para sí mismos las autoinstrucciones e instrucciones específicas de cada tarea mientras el terapeuta resuelve la tarea con autoinstrucciones encubiertas.

5) Los niños realizan la tarea utilizando el lenguaje interno (autoinstrucciones e instrucciones específicas encubiertas).

Las sesiones se llevaron a cabo en una sala especialmente habilitada para tal fin y se fijó el día y hora de entrenamiento para cada uno de los grupos establecidos, día y hora que se mantuvieron constantes a lo largo del tratamiento. Las sesiones se iniciaron con la llegada de todos los niños del grupo y se utilizó un cartel de autoinstrucciones colocado en un lugar visible, una pizarra frente a los niños y cada niño tenía delante el cuaderno que contiene las fichas de trabajo del programa para descifrar instrucciones escritas de Orjales (2002b). 


\section{Diseño y análisis de datos}

En la primera semana, en la fase del pretest, todos los participantes fueron evaluados con el Test de emparejamiento de figuras conocidas (MFF-20) cuyo objetivo fue medir el estilo cognitivo impulsivo. Posteriormente los niños participaron en el entrenamiento cognitivo autoinstruccional aplicado en 30 sesiones grupales realizadas de forma interdiaria con una duración de 60 minutos por cada sesión. Luego, en la fase del posttest, los participantes fueron evaluados nuevamente con el MFF-20.

Las puntuaciones del estilo cognitivo impulsivo antes y después del entrenamiento fueron comparados con la prueba estadística T de Student en el Programa SPSS 15.0 (logicial especializado en estadística). Los valores $p<0.05$ fueron considerados estadísticamente significativos, es decir estructurales e independientes del azar del muestreo.

\section{Resultados}

El análisis pretest-posttest se presenta en la Tabla 1 y refleja una disminución estadísticamente significativa en el percentil impulsividad $(t=7,83 ; p<.05)$ y en el número de errores $(\mathrm{t}=8,31 ; \mathrm{p}<0.05)$ y un aumento estadísticamente significativo en la latencia $(t=-6,74 ; p<.05)$. Las diferencias estadísticamente significativas entre el pretest y el posttest demuestran la efectividad del entrenamiento autoinstruccional para reducir el estilo cognitivo impulsivo de los niños con TDAH.

Tabla 1. Análisis de la prueba $t$ antes y después del entrenamiento autoinstruccional

\begin{tabular}{|c|c|c|c|c|c|c|c|c|}
\hline & & \multicolumn{3}{|c|}{ Pre test } & \multicolumn{3}{|c|}{ Post test } & \multirow{2}{*}{$\begin{array}{c}\text { Pretest- } \\
\text { Posttest } \\
\mathrm{t}\end{array}$} \\
\hline Prueba & & Nro. & Media & Dt & Nro. & Media & $\mathrm{Dt}$ & \\
\hline \multirow[t]{3}{*}{$\begin{array}{c}\text { MFF- } \\
20\end{array}$} & $\begin{array}{l}\text { Percentil impulsivi- } \\
\text { dad }\end{array}$ & 10,00 & 59,30 & $(23,25)$ & 10,00 & 1,70 & $(0,68)$ & $7,83^{*}$ \\
\hline & Errores & 10,00 & 65,0 & $(48,12)$ & 10,00 & $-140,1$ & $(61,46)$ & $8,31 *$ \\
\hline & Latencia & 10,00 & 10,80 & $(64,01)$ & 10,00 & 297,0 & $(118,08)$ & $-6,74 *$ \\
\hline
\end{tabular}




\section{Discusión y Conclusiones}

El objetivo del estudio fue determinar el efecto del entrenamiento cognitivo autoinstruccional en el estilo cognitivo impulsivo de los niños con TDAH. Los resultados demuestran que el estilo cognitivo impulsivo, característico del TDAH, disminuye significativamente después de la aplicación del entrenamiento cognitivo autoinstruccional basado en la teoría de Meichenbaum y Goodman (1971), el esquema de autoinstrucciones ampliado por Orjales (2007) y el procedimiento de Meichenbaum (1977) para enseñar las autoinstrucciones.

Meichenbaum y Goodman (1971), Kendall y Wilcox (1980) encontraron también efectividad del entrenamiento cognitivo autoinstruccional para reducir la impulsividad. Además, de forma similar a esta investigación, otros estudios (Arco, Fernández e Hinojo, 2004; Calderón, 2001) utilizaron el esquema de Meichenbaum y Goodman y obtuvieron también resultados positivos del entrenamiento autoinstruccional para reducir la impulsividad cognitiva evaluada con el MFF-20 en niños diagnosticados con TDAH.

La importancia de esta investigación radica en que ha puesto en práctica un programa para solucionar un problema cognitivo que afecta a los niños con TDAH en su desenvolvimento en el aula y en el hogar. A partir de los resultados, se concluye que el entrenamiento autoinstruccional reduce la impulsividad cognitiva. Entonces, los niños con el trastorno aprenden a utilizar estrategias reflexivas para resolver tareas y disminuyen la tendencia de responder al azar, de forma precipitada y con errores y sin percibir la información completa, conductas que caracterizan al estilo cognitivo impulsivo. A su vez, la utilización de las estrategias reflexivas para resolver tareas, podría mejorar el rendimiento académico de los niños con TDAH. Por esta razón el entrenamiento autoinstruccional debe aplicarse en el aula.

En la presente investigación, los niños recibieron el entrenamiento autoinstruccional en una sala especialmente implementada para tal fin. De aplicarse en el aula, se recomienda usar el mismo procedimiento, pero debe existir previo un entrenamiento a los profesores. En mi localidad, Arequipa (Perú), los niños con TDAH y sin ninguna comorbilidad asisten a los colegios regulares y se hace imprescindible un manejo efectivo del maestro para atender sus necesidades educativas. 
Existen investigaciones (Arco, Fernández e Hinojo, 2004; Miranda, Soriano, Presentación y Gargallo, 2000) que utilizan el entrenamiento autoinstruccional en el aula y acompañan esta intervención con otras técnicas cognitivo conductuales (entrenamiento a padres y maestros, modificación de conducta, técnicas de autocontrol, autoevaluación, modelado, reforzadores, entrenamiento en habilidades sociales, etc.) y como resultado los niños con TDAH han progresado en la disminución de los síntomas del trastorno y las conductas problema en el aula.

En esta investigación, el entrenamiento cognitivo autoinstruccional ha dado buenos resultados en la reducción de la impulsividad cognitiva a través de la enseñanza de una secuencia de pensamientos más organizados y reflexivos denominados autoinstrucciones (Primero miro y digo todo lo que veo, ¿qué és lo que tengo que hacer?, ¿cómo lo voy a hacer?, tengo que estar muy atento (y ver todas las posibilidades de respuesta), ¡ya puedo hacerlo!, no me ha salido bien ¿por qué? (repaso todos los pasos), ah, ¡era por eso! bien la próxima vez no cometeré ese error). Las autoinstrucciones permiten a los niños reemplazar pensamientos inapropiados o desorganizados respecto de la tarea, regular su conducta y reducir la impulsividad.

El entrenamiento autoinstruccional es utilizado para ayudar a los estudiantes con dificultades en la impulsividad. Por ejemplo, un niño con TDAH que responde las preguntas del maestro sin esperar su turno, puede ser entrenado a responder silenciosamente para sí mismo, sin interrumpir a la clase, o decirse a sí mismo de forma silenciosa "yo sé la respuesta pero puede esperar mi turno" (Shillingford, Lambie y Walter, 2007). Las autoinstrucciones ayudan a las personas con TDAH a adoptar una actitud mas sistemática y productiva hacia las tareas y la resolución de problemas (National Institute for Health and Clinical Excellence, 2009, citado por Serrano-Troncoso, Guidi y Alda-Diez, 2013).

A pesar de los resultados positivos del tratamiento cognitivo autoinstruccional en la disminución del estilo cognitivo impulsivo del TDAH coincido con Orjales (2007) en sostener que el entrenamiento cognitivo autoinstruccional es una técnica útil pero sólo una pequeña parte del tratamiento multimodal que requiere la población de niños con TDAH y que comprende un programa de acción familiar, un programa de intervención escolar y un programa de tratamiento específico dirigido a la persona diagnosticada con el trastorno; este último 
comprende tratamiento farmacológico y un programa de intervención no sólo cognitivo sino también conductual y emocional.

El tratamiento intensivo y multidisciplinario ayuda a los niños con TDAH a afrontar sus dificultades (Miranda y Presentación, 2000). La práctica clínica demuestra que la combinación de diversas técnicas, articuladas en un modelo de intervención coherente, propicia la aparición de mejoras clínicas significativas en mucha mayor medida que el uso aislado de algunas de ellas (Mas, 2009) y deben abarcar tanto al niño, familia y colegio (Abad, Ruiz, Moreno, Herrero y Suay, 2013). La combinación de intervenciones farmacológicas y psicoeducativas para el tratamiento del TDAH están vigentes en la comunidad científica y profesional (Lavigne y Romero, 2010).

Un aspecto relevante en la investigación son algunas características de las familias de la localidad de Arequipa (Perú). Algunos padres de familia consideran que el trastorno es producto de la mala educación que los niños han recibido; además, algunos prefieren las intervenciones psicopedagógicas en lugar de tratamiento farmacológico. Por lo tanto es necesaria la intervención psicoeducatica con los padres acerca de los síntomas diagnósticos del trastorno, la etiología, evolución y tratamiento.

Al respecto, Duda (2005, pág. 43) manifiesta lo siguiente: "Los niños con TDAH no son necesariamente malcriados sino que muchas veces no pueden controlar el impulso de hablar (aunque la maestra esté dictando clase), el impulso de moverse (aunque estén en un examen), el impulso de tener un juego (aunque lo esté usando el amigo), etc". Los niños con TDAH no pueden resistir la tentación de jugar en vez de terminar las tareas escolares porque tienen déficit en el control inhibitorio, es decir tienen dificultad para activar información específica e inhibir respuestas automáticas innecesarias (Sánchez-Pérez y González-Salinas, 2013).

Una de las limitaciones del estudio es el tamaño pequeño de la muestra. Sin embargo, los resultados obtenidos son consistentes con estudios previos. Sugiero replicar la presente investigación en una muestra más grande. Además, a la luz de los resultados positivos obtenidos, queda en siguiente línea investigar si estos resultados se mantienen 6 meses o 1 año después de finalizado el entrenamiento. 
Finalmente, hay que continuar con los esfuerzos para brindar intervenciones especializadas, individualizadas o hechos a la medida de las necesidades, potencialidades y problemas de cada niño con TDAH. Asimismo se debe promover un trabajo multidisciplinario que combine distintos enfoques (médico, psicológico, educativo) en la atención que se brinda a los niños con el trastorno.

\section{Referencias}

Abad, L., Ruiz, R., Moreno, F., Herrero, R. y Suay, E. (2013). Intervención psicopedagógica en el trastorno por déficit de atención/hiperactividad. Revista de Neurología, 57(Extra 1), 193-203. Recuperado de http://dialnet.unirioja.es/servlet/articulo?codigo=4719643

American Psychiatric Association (2013). DSM-V. Diagnostic and statistical manual of mental disorders. 5th. Ed. Arlington, VA: American Psychiatric Association.

Arco, J. L., Fernández, F.D. e Hinojo, F. J. (2004). Trastorno por déficit de atención con hiperactividad: intervención psicopedagógica. Psicothema, 16(3), 408-414. Recuperado de http://www.psicothema.com/pdf/3011.pdf

Bornás, X., Servera, M., Serra, F. y Escudero, J.T. (1990). El tratamiento de la impulsividad infantil: Autoinstrucciones versus solución de problemas. Estudios de Psicología, 43, 61-71. doi: 10.1080/02109395.1990.10821143

Cairns, E.D. y Cammock, J. (2005). Test de emparejamiento de figuras conocidas-20. MFF20. Madrid: TEA.

Calderón, C. (2001). Resultados de un programa de tratamiento cognitivo-conductual para niños con trastorno por déficit de atención con hiperactividad. Anuario de Psicología, 32 (4), 79-98. Recuperado de:

http://www.raco.cat/index.php/AnuarioPsicologia/article/view/61693/88460

Clariana, M. (1993). Reflexividad-impulsividad y estrategias cognitivas. Revista de Psicología General y Aplicada, 46(2), 209-212. Recuperado de: http://fundacioncadah.org/j289eghfd7511986_uploads/20120607_j2mM1nYvFy04TZ 4uRY7R_0.pdf

Duda, B. (2005). El déficit de atención y la hiperactividad en el aula. Signo educativo, 136, 42-43. 
Fernández-Mayoralas, D.M., Fernández-Jaén, A., García-Segura, J.M. y Quiñones-Tapia, D. (2010). Neuroimagen en el trastorno por déficit de atención/hiperactividad. Revista de Neurología, 50(3), 125-133. Recuperado de:

http://dialnet.unirioja.es/servlet/articulo?codigo $=4230526$

Gilliam, J. (1995). Test del desorden por déficit de atención e hiperactividad. Un método para identificar personas con ADHD. Austin: Pro-ed.

Kagan, J., Rosman, B.L., Day, D., Albert, J. \& Phillips, W. (1964). Information processing in the child: Significance of analytic and reflective attitudes. Psychological Monographs: General and applied, 78(1), 1-37. doi: 10.1037/h0093830

Kendall, P.C. \& Braswell, L. (1993). Cognitive-hebavioral training for impulsive children. 2nd. Ed. New York: The Guilford Press.

Kendall, P.C. \& Wilcox, L.E. (1980). Cognitive behavioral treatment for hyperactivity: concrete versus conceptual training in non-self-controlled problem children. Journal of Consulting and Clinical psychology, 48(1), 80-91. doi: 10.1037/0022-006X.48.1.80

Ketzer, C.R., Gallois, C., Martinez, A.L., Rohde, L.A. \& Schmitz, M. (2012). Is there an association between perinatal complications and attention-deficit/hyperactivity disorderinattentive type in children and adolescents? Revista Brasileira de Psiquiatría, 34(3), 321-328. doi:10.1016/j.rbp.2012.01.001

Lavigne, R. y Romero, J. F. (2010). Modelo Teórico del Trastorno por Déficit de Atención con Hiperactividad I: Definición Operativa. Electronic Journal of Research in Educational Psychology, 8(22), 1303-1338. Recuperado de: http://www.redalyc.org/articulo.oa?id=293122000017

Larsson, H., Chang, Z., D'Onofrio, B.M. \& Lichtenstein, P. (2014). The heritability of clinically diagnosed attention deficit hyperactivity disorder across the lifespan. Psychological Medicine, 44 (10), 2223-2229. doi:10.1017/S0033291713002493

López, J.A. (2005). Estilos cognitivos reflexivo-impulsivo, flexible-rígido y dependienteindependiente de campo: análisis discriminante de subtipos del trastorno. Psiquiatría.com, 9(2). Recuperado de http://hdl.handle.net/10401/3514

López, J.A., Serrano, I., Delgado, J., De Llano, J.M. Sánchez, M.I. y Alberola, S. (2011). Utilidad de un modelo estadístico de estilos cognitivos en el trastorno por déficit de atención con hiperactividad. Psicothema, 29 (4), 818-823. Recuperado de http://www.psicothema.com/pdf/3961.pdf

López, J.A., Serrano, I., Delgado, J., De Llano, J.M., Alberola, S., Sacristán, A.N., Pérez, I. y Camina, A.B. (2010). Uso del Matching Familiar Figures Test 20 en el diagnóstico de 
niños con trastorno por déficit de atención con hiperactividad. International Journal of Clinical and Health Psychology, 10 (3), 499-517. Recuperado de: http://www.aepc.es/ijchp/articulos_pdf/ijchp-363.pdf

Mas, C. (2009). El TDAH en la práctica clínica psicológica. Clínica y Salud, 20(3), 249259. Recuperado de http://scielo.isciii.es/pdf/clinsa/v20n3/v20n3a06.pdf

Mateo, V. F. (2006). Recursos para el diagnóstico psicopedagógico del TDAH y comorbilidades. Electronic Journal of Research in Educational Psychology, 4(1), 623-642. Recuperado de http://www.redalyc.org/articulo.oa?id=293121929009

Meichenbaum, D. (1977). Cognitive-behavior modification. An integrative approach. New York: Plenum Press.

Meichenbaum, D. \& Goodman, J. (1971). Training impulsive children to talk to themselves: A means of developing self-control. Journal of Abnormal Psychology, 77(2), 115-126. doi: $10.1037 / \mathrm{h} 0030773$

Miranda, A. \& Presentación, M.J. (2000). Effects of cognitive-behavioural treatment in aggressive and nonaggressive children with Attention Deficit Hiperactivity Disorder. Significant clinical change. Infancia y aprendizaje, 23(4), 51-70. doi: $10.1174 / 021037000760087775$

Miranda, A. y Soriano, M. (2010). Tratamientos psicosociales eficaces para el trastorno por déficit de atención con hiperactividad. Información psicológica, 100, 100-114.

Miranda, A., Soriano, M., Presentación, M.J. y Gargallo, B. (2000). Intervención psicoeducativa en estudiantes con trastorno por déficit de atención con hiperactividad. Revista de Neurología Clínica, 1, 203-216. Recuperado de http://www.jmunozy.org/files/9/Necesidades_Educativas_Especificas/tdah/documento s/Intervencion_psicoeducativa_en_alumnos_con_TDAH_Ana_Miranda_.pdf

Orjales, I. (2002a). Déficit de atención con hiperactividad: Manual para padres y educadores. Madrid: CEPE.

Orjales, I. (2002b). Programa de entrenamiento para descifrar instrucciones escritas. Especialmente indicado para niños y niñas impulsivos y con déficit de atención con hiperactividad (TDAH). Madrid: CEPE.

Orjales, I. (2007). El tratamiento cognitivo en niños con trastorno por déficit de atención con hiperactividad (TDAH): revisión y nuevas aportaciones. Anuario de Psicología Clínica y de la Salud, 3, 19-30.

Orjales, I. y Polaino-Lorente, A. (1992). Estilos cognitivos e hiperactividad infantil: los constructos dependencia-independencia de campo perceptivo e impulsividad-reflexividad. 
Bordón, 44(4), 421-430. Recuperado de: http://dialnet.unirioja.es/servlet/articulo?codigo $=54427$

Polaino-Lorente, A.; Avila, C.; Cabanyes, J.; García, D.; Orjales, I. y Moreno, C. (1997). Manual de hiperactividad infantil. Madrid: Unión Editorial.

Ramiro, P., Navarro, J.I., Menacho, I. y Aguilar, M. (2010). Estilo cognitivo reflexividadimpulsividad en escolares con alto nivel intelectual. Revista latinoamericana de Psicología, 42 (2), 193-202. Recuperado de: http://www.redalyc.org/articulo.oa?id=80515381002

Rivera-Flores, G.W. (2013). Etiología del trastorno por déficit de atención e hiperactividad y características asociadas en la infancia y la niñez. Acta de Investigación Psicológica, 3(2), 1079-1091. Recuperado de http://www.scielo.org.mx/pdf/aip/v3n2/v3n2a3.pdf

Sánchez-Pérez, N. y González-Salinas, C. (2013). Ajuste Escolar del Alumnado con TDAH: Factores de Riesgo Cognitivos, Emocionales y Temperamentales. Electronic Journal of Research in Educational Psychology, 11(3), 527-550. doi: http://dx.doi.org/10.14204/ejrep.30.12189

Serrano-Troncoso, E., Guidi, M. y Alda-Diez, J.A. (2013). ¿Es el tratamiento psicológico eficaz para el trastorno por déficit de atención con hiperactividad (TDAH)? Revisión sobre los tratamientos no farmacológicos en niños y adolescentes con TDAH. Actas Españolas de Psiaquiatría, 41(1), 44-51. Recuperado de http://actaspsiquiatria.es/repositorio/15/81/ESP/15-81-ESP-44-51-322427.pdf

Shillingford, M.A., Lambie, G.W. y Walter, S.M. (2007). An integrative, cognitivebehavioral, systemic approach to working with students diagnosed with attention deficit hyperactive disorder. Professional School Counseling, 11(2), 105-112. doi: 10.5330/PSC.n.2010-11.105

Weschler, D. (2005). WISC IV: La escala de inteligencia para edad escolar. 4ta. ed. Madrid: TEA. 